und ist Vergesellschaftung fragmentarisch verlaufen. Statt von einer fragmentierten Gesellschaft wäre es daher zweckmäßiger, von gesellschaftlichen Fragmenten $\mathrm{zu}$ sprechen.

Zweitens eröffnet der Begriff der Gemeinschaft die Möglichkeit von Zuschreibungen, die ethnopolitische Kategorien in ihrer empirisch auftretenden Form, anhand real existierender, Klan-artiger Netzwerke beschreibt, ohne sie zu essenzialisieren. Denn natürlich sind im gegenwärtigen politischen Kontext Zuschreibungen wie Dinka, Nuer oder, wie beim derzeitigen Oberkommandierenden der SSPDF, Acholi, von Belang. Die beständige Reflexion des fluiden, in permanenter Rekonstruktion befindlichen Charakters derartiger Zuschreibungen ist aber unumgänglich. Die folgenden beiden Vignetten, die Ausschnitte aus der ethnopolitischen Konfliktlandschaft bieten wollen, unterstreichen das in aller Deutlichkeit.

\title{
Dinka-Dominanz?
}

Das Narrativ einer angeblichen Dominanz der Dinka, vor allem der DinkaGemeinschaften aus Bahr el-Ghazal, ist ein langjähriger Vorhalt gegenüber der SPLM/A. Doch das Narrativ ist älter. Schon während der Zeit des Addis Ababa Agreement und der Auseinandersetzungen zwischen der von Equatorians dominierten Anya-nya und den den Süden repräsentierenden Politikern in Khartum, die zumeist aus den nördlichen Regionen stammen, kommt es erstmals zu derartigen Vorwürfen (Rolandsen und Daly, 2016: 95). In der späteren Phase des Krieges steht die SPLM/A-Führung nahezu permanent unter dem Verdacht, in erster Linie eine Dinka-Agenda zu verfolgen. Deren ethnopolitische Komposition rund um den aus Jonglei stammenden Twic Dinka John Garang oder den aus Gogrial stammenden Awan-Chan Dinka Salva Kiir trägt zu diesem Bild bei. Die Spaltung der Organisation durch den NasirSplit im Jahr 1991, während der der Dok Nuer aus Unity State, Riek Machar, versucht, eine ethnopolitisch argumentierte Nuer-Shilluk-Allianz gegen die Dinka-Führung zu etablieren, verstärkt und verstetigt dieses Image. Nachdem die im Zuge des Nasir-Split aufbrechenden Spannungslinien in den folgenden Jahrzehnten nicht mehr transzendiert werden konnten, ist die Einstellung der Nuer-Gemeinschaften zur heutigen SPLM überwiegend distanziert.

Diese Distanziertheit darf nicht darüber hinwegtäuschen, dass es Machar und seinen verschiedenen organisatorischen Vehikeln nie gelingt, eine ethno- 
politische Nuer-Allianz gegen die SPLM zu etablieren. Ebenso ist, auf der anderen Seite, Salva Kiir wiederholt mit gefährlichen Herausforderungen und Spaltungsversuchen ambitionierter Dinka konfrontiert. Der ehemalige Generalstabschef Paul Malong liefert dafür das vielleicht prominenteste Beispiel. Ethnopolitik ist trotzdem ein wesentlicher Faktor. Dies zeigen nicht wenige der jüngsten Postenbesetzungen in höchsten Regierungsämtern, die durch das Interesse der Herstellung eines ethnopolitischen Ausgleichs oftmals besser zu erklären sind als durch politische Transaktionen oder gar die spezifischen Fähigkeiten der Ernannten. Die Beförderung von Johnson Juma Okot, einem Equatorian, zum Generalstabschef ist ein derartiges Beispiel.

Bei einer Reihe einflussreicher politischer Figuren zeigt sich, dass sich die SPLM-Spitze keineswegs nur auf Dinka verlässt. Der Jikany Nuer Taban Deng Gai, der nun eine regierungstreue Minderheitsfraktion der SPLM/A-IO vertritt, verdankt seine Position als derzeitiger Vizepräsident, wie zuvor gezeigt, in erster Linie seiner spezifischen politischen Funktionalität und seinem nach Jahrzehnten des politischen Spiels enormen persönlichen Netzwerk. Ebenso spielen einige Nuer wichtige Rollen im derzeitigen Regime, ohne dass sie eine Verbindung zur Opposition bereitstellen würden. Der im vorangegangenen Kapitel vorgestellte Tut Kew Gatluak verkörpert ein solches Beispiel. Auch Riek Gai Kok, Luo Nuer aus Akobo, der lange als Gesundheitsminister amtierte und im Gegenzug dazu seine exzellenten Beziehungen in Khartum einbrachte, ist ein solches Beispiel. Um die ethnopolitischen Ausgleichsbemühungen von Kiir hat sich über die Zeit eine Reihe an urbanen Legenden gebildet: "As one former official put it, if you want an appointed position, syou go to Salva while he is drinking, if anyone refuses you say [with pretend anger] »It is because I am Nuer« and he signs».« (Stringham und Forney, 2017: 191)

Zugleich kann eine Mobilisierung entlang ethnopolitischer Linien nicht bestritten werden. Dabei spielen Diaspora-Gruppen eine nicht zu unterschätzende Rolle. Die Mobilisierung der Dinka ist auf nationaler Ebene gut organisiert. Als zentrales Instrument fungiert das Jieng Council of Elders (JCE), das mit seinen etwa 45 Mitgliedern, die die verschiedenen Regionen des Landes repräsentieren, direkten Druck auf den Präsidenten ausübt. Das genaue Gründungsdatum des JCE ist unbekannt, es dürfte um das Jahr 2013 liegen. Klar ist, dass es auf einem Abwehrnarrativ aufbaut. ${ }^{1}$ Dies bestätigen gegenwärtige 
Mitglieder in Interviews, die von Diaspora-Gruppen verbreitet werden. ${ }^{2} \mathrm{Pe}-$ ter Adwok Nyaba (2019: 152) versteht die Formierung des JCE nicht zuletzt vor dem Hintergrund eines "surge in Dinka ethnic nationalism, with its ideology of hegemony and domination«. Gleichzeitig betont er dessen wichtige Funktion in der Organisation eines ethnopolitischen Diskurses, da es »bringing together in organizational and ideological unity Dinka intellectuals from the different Dinka sections, including Abyei«.

Geleitet wird das JCE seit seiner Gründung durch den ehemaligen Präsidenten des Obersten Gerichtshofes, Ambrose Riiny Thiik, dem eine zentrale Rolle bei der Mobilisierung der von Paul Malong kontrollierten Dinka-Miliz Mathiang Anyoor nachgesagt wird. Überhaupt kann Paul Malong als zentraler politischer Verbündeter des JCE gesehen werden. In Fragen wie der Anzahl und Gestaltung der Gliedstaaten vertraten JCE und Malong die gleiche Position. Ebenso zeigten sich beide ablehnend gegenüber einem Friedensvertrag mit der SPLM/A-IO und deren Einbeziehung in eine Übergangsregierung. Peter Nyaba (ebd.) sieht das oftmalige Eingehen von Kiir auf Forderungen des JCE als seine größte persönliche Schwäche. Kiir »acquiesced and implemented JCE policies. The Jieng's political-military and business elite had twin objectives in the nascent Republic of South Sudan: to install absolute medieval-like Jieng power and to achieve the economic empowerment of its members. « Diese Einschätzung vernachlässigt jedoch, dass sich Kiir in einigen entscheidenden Momenten klar gegen das JCE gestellt hat: etwa bei der Entlassung von Paul Malong oder der Rückkehr von $32 \mathrm{zu} 10$ Gliedstaaten, einer Entscheidung, die das JCE ausdrücklich ablehnte.

Der tatsächliche Einfluss und die tatsächlichen Interessen des JCE bleiben unbestimmbar. Einiges spricht dafür, dass sich sowohl Einfluss als auch unmittelbare Interessen in einem Prozess der permanenten Aushandlung befinden. Aufgrund der sichtbaren Entscheidungen der südsudanesischen Regierung und der einsehbaren Äußerungen des JCE lässt sich die immer wieder auftauchende Verschwörungstheorie, wonach das JCE in Wahrheit das Land regiere, entkräften. Auf der anderen Seite kann wenig Zweifel daran bestehen, dass das JCE versucht, gemeinsame Interessen der Dinka zu identifizieren, verweist, dass es etwa die Nuer-Abspaltungen seit Nasir seien, die das JCE überhaupt erst zusammengebracht hätten. https://www.sbs.com.au/language/english/audio/weare-protecting-the-interest-of-jieng-aldo-ajou-explained-the-role-of-jce [20-01-2021]. 
zu formulieren und durchzusetzen. Das ist klassische Ethnopolitik, die phasenweise an offenen Rassismus grenzt.

Ein Beispiel für die Funktionsweise solcher Einflussnahmen liefert die Ende des Jahres $2020 \mathrm{zu}$ heftigen politischen Auseinandersetzungen führende Nominierung des Shilluk Johnson Olonyi von der SPLM/A-IO zum Gouverneur in Upper Nile State, über die im folgenden Kapitel noch mehr zu lesen sein wird. Formell ist weder das JCE noch irgendeines seiner Mitglieder in der Position, Olonyis Ernennung zu verhindern. Zwar haben einige der Jieng Elders politische Funktionen inne, etwa als Parlamentsabgeordnete im Oberhaus, sie sind jedoch weder formell noch informell als Einzelpersonen in der Lage, auf offizieller Ebene Einfluss auf politische Entscheidungen auszuüben. Es ist diese Konstellation, die vermutlich überhaupt erst die Formierung eines Council of Elders notwendig gemacht hat.

Ein Aspekt, der die Jieng Elders immer umtreibt, ist die Frage von Land und dessen Kontrolle. Ein gutes Beispiel dafür ist JCE-Leiter Ambrose Riiny. Weit davon entfernt, Eigeninteressen gegenüber einer ethnopolitisch geschweißten Dinka-Allianz zurückzustellen, spielt er eine wesentliche Rolle bei Auseinandersetzungen innerhalb der Dinka-Gemeinschaften in seinem Heimatgebiet Gogrial, offenkundig mit dem Ziel, einen eigenen Gliedstaat für seine Apuk Dinka zu erzwingen.

Auch bei der Kontroverse um eine Ernennung Olonyis bringt das JCE die Frage der Kontrolle des Landes in Upper Nile State zur Sprache. Schließlich war Olonyi selbst während des Bürgerkrieges für die Zurückwerfung von Dinka-Verbänden verantwortlich und, aus Sicht von JCE, an der Wegnahme von Dinka-Land beteiligt. Schon von daher ist die Postenbesetzung für das JCE inakzeptabel. Dem Vernehmen nach hatten Mitglieder des JCE die Mobilisierung von Dinka-Milizen in der Region und die umgehende bewaffnete Bekämpfung Olonyis angekündigt, wäre es zu seiner Ernennung gekommen. Diese Episode zeigt Verschiedenes: Erstens geht es dem JCE weniger um eine stringent identitätspolitisch argumentierte, de facto rassistische Herrschaft der Dinka über andere Gemeinschaften. Vielmehr stehen konkrete politischökonomische Interessen im Vordergrund, die für zumindest einige der Mitglieder auch persönlichen Nutzen beinhalten. In der Artikulation seiner Forderungen gleicht das JCE einer Lobbyorganisation, die ihre Verankerung in den von Dinka-Gemeinschaften bewohnten Regionen des Landes und ihre Verbindung zu bewaffneten Dinka-Milizen als Hebel zur Durchsetzung ihrer Forderungen einsetzt. 
Von einer beständigen Interessenübereinstimmung zwischen der Präsidentschaft, anderen Dinka in der politischen Elite, wie etwa dem als faktischer Leiter des NSS zweitmächtigsten Mann des Staates, Akol Koor Kuc, und dem JCE kann keine Rede sein. Vorhaltungen eines geplanten »Ethnozids«, wie ihn vor Zeiten eine aus Kanada stammende Anthropologin in einem von Diaspora-Gruppen im Umlauf gebrachten Pamphlet an die Wand malte, entspringen aller Wahrscheinlichkeit nach den Fantasien der Verfasserin. Dies heißt jedoch nicht, dass Ethnopolitik nicht mörderische Konsequenzen nach sich ziehen kann. Verschiedene Kampfepisoden während des südsudanesischen Bürgerkrieges weisen eindeutig genozidale Elemente auf. Diese lassen sich jedoch, selbst angesichts des in einigen Fällen offenbar geplanten Charakters, ebenso plausibel auf das Zusammenfallen der langfristig etablierten ethnopolitischen Diskurse mit der Hitze von Gefechtssituationen zurückführen und liefern mithin keinen eindeutigen Beleg auf genozidale Absichten.

Nichtsdestotrotz ist Vorsicht geboten. Essenzialisierende Analysen, gerade von internationalen Expert:innen, haben das Potenzial, die institutionalisierte Ethnopolitik in einem potenziell fatalen Maß zu befeuern. Egal, wie die jeweilige Analyse ausfällt, sie wird Unterstützer:innen und Verbreiter:innen finden, die versuchen werden, solche Veröffentlichungen direkt in politisches Kleingeld umzumünzen. Vor diesem Hintergrund hat wissenschaftliche Arbeit im Südsudan eine besondere Verantwortung. Unbedachte Veröffentlichungen, so schreiben vier Kolleginnen in Antwort auf die unglückliche Schrift der zuvor erwähnten kanadischen Anthropologin, wären »extremely inflammatory in tense current circumstances«. Daher wären Forschende aus dem Globalen Norden dazu aufgerufen, »[to] must work to the highest ethical and methodological standards in fragile, high-tension situations, especially as our work has the potential to increase risks of harm for South Sudanese colleagues and residents. $\aleph^{3}$

\section{Vielschichtigkeit. Die ethnopolitische Konfliktlandschaft in Jonglei}

Ein zweites Beispiel für die komplexe Wirkung der Konfliktlandschaft der Ethnopolitik findet sich in Jonglei. Die volatile Konstellation zwischen Bor [20-01-2021]. 